\title{
IMPROVING PERFORMANCE OF DEBLOCKING TECHNIQUES USING IMAGE FUSION
}

\author{
Amina Saleem ${ }^{1}$, Azeddine Beghdadi ${ }^{1}$, and Boualem Boashash ${ }^{2,3}$ \\ 1.L2TI-Institute Galilee, Universite Paris 13, Villetaneuse, France \\ 2.Qatar University College of Engineering, Doha Qatar \\ 3. The University of Queensland, Centre for Clinical Research, Brisbane, Australia
}

\begin{abstract}
Block based transform coding is the most popular technique for image and video compression. However, images compressed using block based algorithms exhibit blocking which is one of the most annoying artifacts in compressed images and video. Many deblocking algorithms have been proposed to improve the quality of the reconstructed image. Although the deblocking operation improves the image appearance by smoothing the block discontinuities, a loss of information also occurs during this process. As a consequence, there is a need to develop methods to reduce the blocking artifacts while minimizing the information loss. We propose a novel fusion based technique to minimize the loss of useful information which may result from the deblocking operation. The loss of key image information can be reduced by fusing a deblocking result and the compressed image.
\end{abstract}

Index Terms-Image enhancement, Image decomposition, Image fusion, Discrete wavelet transforms

\section{INTRODUCTION}

Block transform coding is one of the most popular approaches for image and video compression. It is used in compression standards such as JPEG, MPEG and H.26x due to its excellent energy compaction capability and low hardware complexity. However at high compression ratios, the block based coding methods produce blocking artifacts that appear as artificial discontinuity between adjacent blocks. These are a result of independent processing of blocks and the quantization of coefficients within each block [1]. The Mach phenomenon further increases the perceived contrast of adjacent regions [2]. Thus the visibility of the blocking effect is amplified due to the horizontal and vertical sensitivity of the HVS.

The blocking artifact has been widely studied and many methods for measuring and reducing these artifacts have been proposed in the literature. Most post-processing techniques for removing blocking artifacts filter images in the spatial [6] or the transform domains (e.g., discrete cosine transform (DCT) domain [8] or the wavelet domain [7]). The filters used have some low pass characteristics so the deblocking procedure is actually a smoothing operation. Therefore, while deblocking reduces the blocking artifacts, critical high frequency information is also lost during the process.

Most post filtering methods for deblocking are based on the assumption that blocking artifacts occur at predictable coordinates (generally some multiples of 4,8 or 16). However in compressed video, such as MPEGx or H264x and even in the new and efficient standard HEVC, blocking effect can appear anywhere. This is mainly due to motion estimation and compensation errors. Some adhoc solutions have been proposed in the HEVC scheme [14]. For a generalized post filtering method, it is desirable not to assume that blocking artifacts occur at known positions. The advantage of the filtering based deblocking techniques is that low pass filtering in a local region of each pixel can be performed without considering predicted coordinates of blockiness [6]. Moreover, these don't require additional information to be transmitted or any additional operations on the coder side. The biggest drawback of such techniques (which is common to all low pass filtering approaches) is unnecessary blurring of the actual image edges.

We can summarize from the above discussion that conventional filtering based deblocking algorithms reduce the blocking artifacts but result in the loss of important high and mid-frequency information. The major challenge is how to effectively smooth the blocking artifacts without blurring image details. This is however a conflicting goal to achieve i.e. for areas with sharp transitions (texture), even a small smoothing region can result in loss of edge information. On the other hand for plain image regions, a small smoothing fails to effectively eliminate the blockiness. A variety of methods aim to reduce the blocking while minimizing the loss of original image information loss [10], [11]. We propose a novel fusion based method to minimize the loss of key information which may result during the deblocking operation. The main idea is to minimize the loss of image information by fusing the deblocked result 
(deblocked using any deblocking technique) and the compressed images. It is interesting to note that the proposed methodology can be used to improve the performance $^{1}$ (by minimizing the loss of information) of any image and video deblocking algorithm. It can be particularly useful for filtering based methods where prior information about the occurrence of the blocking artifacts is not available.

The performance of the method is evaluated using quantitative measures and subjective evaluation. The results are evaluated objectively using a full reference image quality measure, the PSNR-HVS-M [15]. It has been shown recently that PSNR-HVS is well adapted to evaluate the quality of JPEG compressed images [16]. The perceptual quality of the results is estimated by using a perceptual blocking measure proposed in [12]. This measure estimates the perceptual quality taking in to account both the blocking and blur present in the compressed images.

The paper is structured as follows: The theoretical fundamentals and the mathematical description are presented in section 2. The results are discussed in Section 3. Finally, we conclude and propose some perspectives for future work.

\section{PROPOSED FUSION METHOD}

The implementation of the proposed fusion method is based on the characteristics of the blocking artifacts and the deblocking techniques.

- The blocking artifacts manifests as abnormally high frequencies in the horizontal and vertical block boundaries of the block coded (BCD) images. Therefore, the high frequency information of the BCD images changes but the medium and the low frequency remains unchanged or less affected (same as that of the image before compression).

- The deblocking filter removes the high frequency discontinuity over the block boundary However, this smoothing operation results in a loss of important textural information as well as the information in the mid-frequency bands.

Based on the above observations we propose to fuse the output of deblocking process and the compressed original images to minimize the information loss during the deblocking operation. This is achieved by integrating the high frequency content of the deblocked image with medium and low frequency information extracted from the compressed image. To fuse images according to the goals outlined above, we need to represent the images such that they are localized in the spatial-frequency domain. We select the 2D discrete wavelet decomposition system with perfect reconstruction [9] as it provides a convenient multi- resolution representation localized both

\footnotetext{
1 Improved performance here refers to a minimization of information loss without affecting the blocking artifact reduction performance of the deblocking technique.
}

in space and spatial-frequency domains. Moreover, the image at each level is decomposed into sub-images containing low and high frequency components. The high frequency sub-images contain fine details in horizontal, vertical and diagonal directions. The blocking artifacts appear as horizontal and vertical edges. This representation is suitable for the present problem as we can define fusion rules for the horizontal vertical and diagonal directions to retain maximum information in the fused image. Let $y_{C}=\left\{y_{C}{ }^{1}, y_{C}{ }^{2}, \ldots y_{C}{ }^{K}, x_{C}{ }^{K}\right\}$ and $y_{D}=\left\{y_{D}{ }^{1}, y_{D}{ }^{2}, \ldots y_{D}{ }^{K}, x_{D}{ }^{K}\right\}$ be the wavelet decomposition of the compressed and the deblocked images, respectively. $x^{K}$ corresponds to the approximation image at the coarsest level $K$ and $y^{k}, k=1, \ldots, K$ are the detail images at different levels. The detail at each level $k$ comprises different frequency band and orientations. $y^{k}$ is composed of $P$ detail images i.e. $y^{k}=$ $\left\{y^{k}(. / 1), \ldots . y^{k}(. / P)\right\} . y^{k}(. \backslash 1), y^{k}(. \backslash 2)$ and $y^{k}(. \backslash 3)$ are the detail images corresponding to the horizontal, vertical and diagonal directions.

The construction of the fusion rule is based on different physical meaning of the approximation and detail coefficients and a priori information about the application. Detail images contain edges and local detail information. The approximation images on the other hand contain some coarse textural information. We observe that the blocking effect is visible in the detail images (corresponding to level $k=1$ ) for the horizontal and the vertical directions of the wavelet decomposition. Therefore, the detail coefficient of the fused image corresponding to the horizontal and vertical directions for level $(k=1)$ should have contribution from the deblocked image while for the diagonal direction the coefficients should be taken from the compressed image (as they are less affected by blocking). Similarly, the coefficients of the composite MRD (multi-resolution decomposition) for levels $(k=2, \ldots K)$ should be taken from the compressed image. These coefficients retain important textural and edge information of the original image (image before compression) which may be lost or affected by the deblocking operation. Mathematically, the fusion rule for each level $\mathrm{k}$ is expressed by:

$$
\begin{gathered}
y_{F}^{k}(. / p)=\left\{\begin{array}{cc}
y_{D}^{k}(. / p) & \text { for } k=1 \text { and } p=1,2 \\
y_{C}^{k}(. / p) & \text { for } k=1 \text { and } p=3
\end{array}\right. \\
y_{F}^{k}=y_{C}^{K} \quad \text { for } k=2 \ldots . . K \\
x_{F}^{K}=x_{C}^{K}
\end{gathered}
$$

The composite image is then obtained by applying the inverse transformation to the composite wavelet representation $y_{F}$. The proposed fusion method can be sumarized as follows:

Step 1: Compute the wavelet decomposition of the compressed $y_{C}$ and the deblocked $y_{D}$ images, respectively.

Step 2: Form composite wavelet coefficient $y_{F}$ using equation 1 . 
Step 3: Obtain the final fused image by applying the inverse transformation to the composite wavelet representation.

\section{RESULTS AND DISCUSSION}

In this section, simulation results for the proposed fusion method are presented. It is worth noticing that the proposed method can be applied to the output of any deblocking technique. We test the results of our method by applying it to the output of some well known and recent deblocking algorithms proposed in [3-7]. The results for the iterative method (gradient map [3] and visibility map [5] method) and non iterative filtering method (Zhai method [4]) are presented here. The iterative filtering methods (gradient map and visibility map method) suppress the blocking artifacts but results in excessive blurring of image details. The Zhai method attempts to find a right region determination scheme for smoothing with each image block and conducts the corresponding post filtering in shifted windows (PSW) [4]. The Zhai method prevents from possible oversmoothing of image details.

We compare the output of each of the deblocking (Zhai, gradient and visibility map) technique and the fused result to assess the improvement achieved by the proposed (fusion based) method. The results are evaluated using quantitative measures as well as visual inspection. The subjective quality of the results is assessed in the ability of the method to improve the perceptual quality of the image in terms of texture and edge information preservation.

As mentioned above, the aim of the proposed method is to minimize the loss of information during the deblocking operation. Therefore, it is logical to use some image fidelity criteria to measure the similarity between the original (uncompressed) and deblocked images. Among the various image fidelity metrics, we select the PSNRHVS-M measure. It has been shown recently that PSNRHVS gives best results (among a set of image quality measures (IQMs)) for the JPEG compressed images [16]. The authors in [16] ranked different IQMs for each type of degradation. The PSNR-HVS is the peak signal to noise ratio (PSNR) taking into account the contrast sensitivity of the human visual system [17]. The PSNRHVS-M further incorporates the in between coefficient contrast masking of DCT basis functions into the PSNRHVS metric [15].

The testing images used in this section are the "Trees", "Peppers" and the "Baboon". The image "Baboon" has lots of details, the "Peppers" image is largely smooth and the "Trees" image is somewhere in between the two images. We have used bi-orthogonal wavelets (to compute the DWT) for two levels of decomposition $(K=2)$. We get similar performance for decomposition levels $K \geq 3$. The reason is that the horizontal and vertical detail coefficients of the deblocked image coresponding to level $k=1$ (which remain the same even for decomposition levels greater than 2) only contribute towards the composite MRD decomposition .
While the rest of the coeeficients of the composite decomposition (diagonal coefficients corresponding to level $\mathrm{k}=1$ and the coefficients for levels $(\mathrm{k}=2, \ldots \mathrm{K})$ ) are taken from the compressed image. The PSNR-HVS$\mathrm{M}$ index for the test images is presented in Table 1. The results in Table 1 show an improvement in PSNR-HVS$M$ values for our method based on fusing a deblocked output and the compressed image.

To measure the perceptual quality of the results we use a measure developed in [13] to predict perceived JPEG quality. The measure incorporates features to measure blocking and blur artifacts and combines them to constitute the perceived JPEG quality measure. This metric is useful to assess the overall blocking and blur performance. The results of the metric for the test images are presented in Table 1.

We provide the visual results of the proposed algorithm through examples in Figs 1, 2 and 3. Figs. 1, 2 and 3 illustrate the obtained results on the test images. Figs. 1(a), 2(a) and 3 (a) show the compressed test image (compressed with a quality factor of $10, \mathrm{~F}_{\mathrm{q}}=10$ ). Figs. 1 (b), 2(b) and 3(b) are the filtered image using the gradient map, Zhai and the visibility map methods, respectively. Figs. 1(c), 2(c) and 3(c) present the results of the proposed method. The results of the proposed method (Fig. 1(c), 2(c) and 3(c)) show an improvement in the texture and edge information when compared to the output of the deblocking algorithms (the Zhai, the gradient map and the visibility map method in this case). Moreover, we notice that for the iterative filtering (gradient map and visibility map) based methods, the proposed fusion methodology (Fig 1(c) and 3(c)) results in a marked improvement in the image edge information (and the image appears less blurred). For better visibility, a region of "Trees" image (Fig. 1) is zoomed and shown in Fig. 4. We can see that the gradient map method (Fig. 4b) over-smoothes (introducing noticeable blur) the image in general. The proposed method reduces the effect of blur as can be seen in Fig. 4c. Indeed, the obtained results confirm an improvement of the texture and edge information which is lost during the deblocking operation.

The objective evaluation supported by visual analysis shows the effectiveness of the proposed method in improving image sharpness and texture information while retaining the deblocking performance.

\section{CONCLUSIONS}

In this paper we propose a methodology to minimize the loss of information resulting from the deblocking operation. This can be achieved by fusing information from the compressed and the deblocked images. Experiments show the promise of image fusion techniques to achieve conflicting goals (good deblocking performance with minimum information loss) which are otherwise difficult to achieve using traditional approaches.

We show an improvement in the performance of the proposed method by means of improved peak signal to 
noise ratio (PSNR-HVS-M index), perceptual blocking measure as well as improved visual quality. The results confirm the efficiency of the method in enhancing perceptual image quality by increasing image detail without affecting the deblocking (block artifact reduction) performance. We will further investigate the performance using larger database (to compare the average PSNR-HVS-M over all images in the dataset) and the effects of the choice of the wavelet on the fusion result. The proposed method can be extended to MPEG, which is the motivation for future work. The idea proposed in this paper can also be extended to minimize information loss occurring during the image enhancement/restoration operations (such as denoising and deringing) by fusing the post-processed and original degraded images.

\section{REFERENCES}

[1] S.A. Karunasekera, and N.G. Kingsbury, "A distortion measure for blocking artifacts in image based on human visual sensitivity," IEEE Transaction on Image Processing, vol. 4, pp.713-724, 1995.

[2] W.T. Welford, "The visual Mach effect," Physics Education, vol. 3, pp. 83-8, 1968.

[3] A.Saleem, A.Beghdadi, A.Chetouani, and B. Boashash, "Iterative blocking artifact reduction based on local contrast information,'IEEE International Symposium on Signal Processing and Information Technology, (ISSPIT), 2011.

[4] G.Zhai, W.Zhang, X.Yang, and W.Lin, "Efficient image deblocking based on post filtering in shifted windows," IEEE Transactions on Circuits and Systems for Video Technology, vol.18, pp. 122-126, 2008.

[5] A. Chetouani, G. Mostafaoui, and A. Beghdadi, "Deblocking method using a perceptual recursive filter, " IEEE International Conference on Image Processing, 2009.

[6] S. Minami, and A. Zakhor, "An optimization approach for removing blocking effects in transform coding," IEEE Transactions on Circuits System Video Technology, vol. 5, pp. $74-82,1995$

[7] I.H. Jang, N.C. Kim, and H.J So, "Iterative blocking artefact reduction using a minimum mean square error filter in wavelet domain," Signal Processing, vol.83, pp. 2607-2619, 2003.

[8] Y. Luo, and R. K. Ward, "Removing the blocking artifacts of block based DCT compressed images," IEEE Transactions on Image Process., vol. 12, pp. 838-842, 2003.

[9] S.G. Mallat, A Wavelet tour of signal processing. Academic Press, San Diego, 1998.

[10] Y. Yang, N. P. Galatsanos, and A. K. Katsaggelos, "Projection based spatially adaptive reconstruction of blocktransform compressed images," IEEE Transactions on Image Processing, vol. 4, pp. 896-908, 1995.

[11] Y. Yang, N. P. Galatsanos, and A. K. Katsaggelos, "Regularized reconstruction to reduce blocking artifacts of block discrete cosine transform compressed images," IEEE Transactions on Circuits and Systems for Video Technology, vol. 3, pp. 421-432,1993.
[12] Z. Wang, H.R. Sheikh, and Alan C. Bovik, "No-Reference Perceptual Quality Assessment of JPEG Compressed Images," IEEE International Conference on Image Processing, vol.1, pp. 477-480, Sept 2002.

[13] https://ece.uwaterloo.ca/ z70wang/research/nr_jpeg_quality/inde x.html

[14] M. Naccari, C.Brites, A.J.Catarina, F.Pereira, "Low complexity deblocking filter perceptual optimization for the HEVC codec,"in the 18th IEEE International Conference on Image Processing, ICIP 2011, pp.737-740.

[15] N.Ponomarenko, F. Silvestri, K. Egiazarian, M. Carli, J. Astola, V. Lukin, "On between-coefficient contrast masking of DCT basis functions", Proceedings of the Third International Workshop on Video Processing and Quality Metrics, VPQM07, Scottsdale, Arizona, USA, 2007.

[16] A.Chetouani, A. Beghdadi, M.Deriche, "Image distortion analysis and classification scheme using a neural approach," 2nd European workshop on Visual Information Processing (EUVIP), pp.183-186, 2010

[17] K. Egiazarian, J. Astola, N. Ponomarenko, V. Lukin, F. Battisti, M. Carli, New full-reference quality metrics based on HVS, CD-ROM Proceedings of the Second International Workshop on Video Processing and Quality Metrics, Scottsdale, USA, 2006.

Table 1: PSNR-HVS-M index and Perceptual JPEG quality score for the test images

\begin{tabular}{|c|c|c|c|}
\hline \multirow{2}{*}{ Image } & Deblocking method & \multicolumn{2}{|c|}{ IQMs } \\
\cline { 3 - 4 } & $\begin{array}{c}\text { PSNR- } \\
\text { HVS-M }\end{array}$ & $\begin{array}{c}\text { JPEG } \\
\text { quality } \\
\text { score }\end{array}$ \\
\hline \multirow{2}{*}{ Trees } & Gradient map method [ 3] & 22.64 & 8.0063 \\
\cline { 2 - 4 } & Proposed method & 26.42 & 8.1464 \\
\hline \multirow{2}{*}{ Peppers } & Zhai method [4] & 28.54 & 6.4225 \\
\cline { 2 - 4 } & Proposed method & 29.17 & 6.6121 \\
\hline \multirow{2}{*}{ Baboon } & Visibility map method [ 5] & 25.81 & 17.82 \\
\cline { 2 - 4 } & Proposed method & 26.21 & 18.02 \\
\hline
\end{tabular}



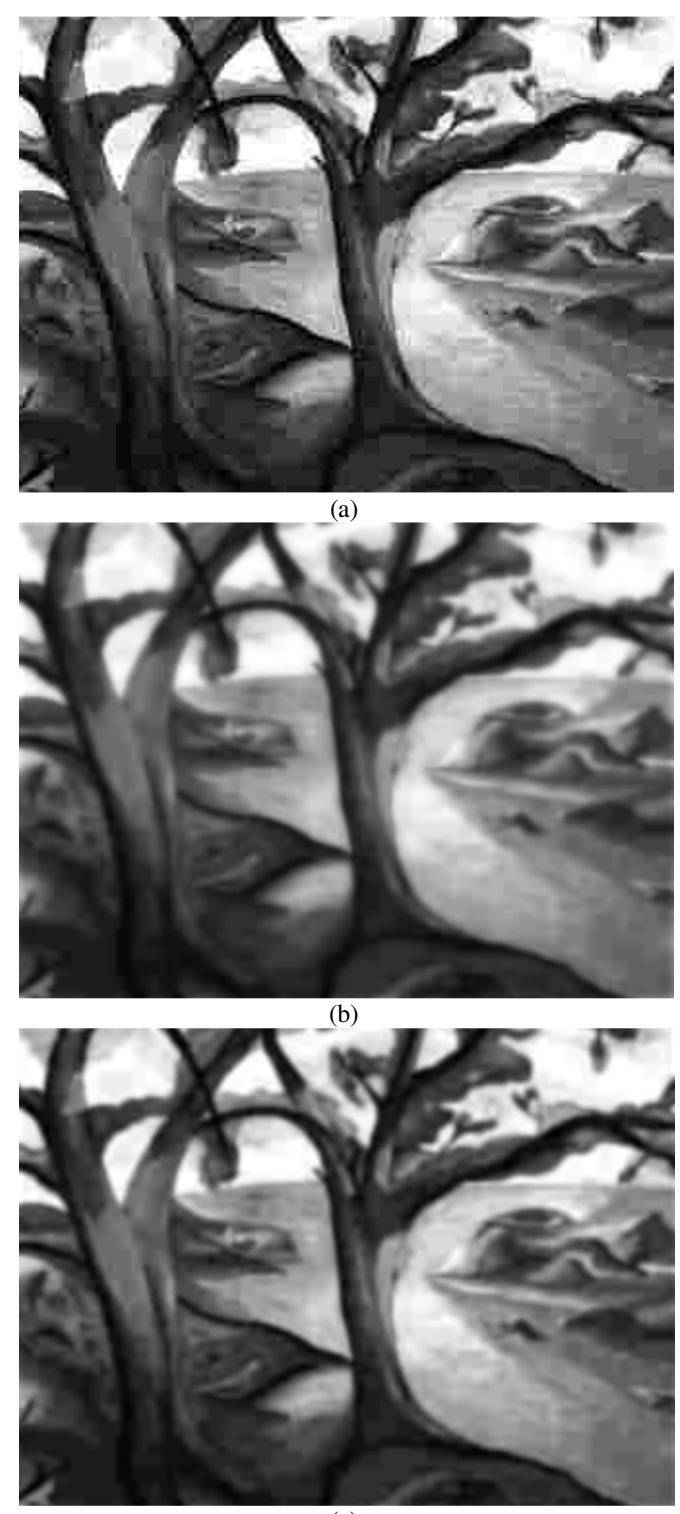

(c)

Figure 1. (a) Compressed "Trees" image with $F_{q}=10$, Deblocked "Trees" image with (b) Gradient map method (c) Proposed method

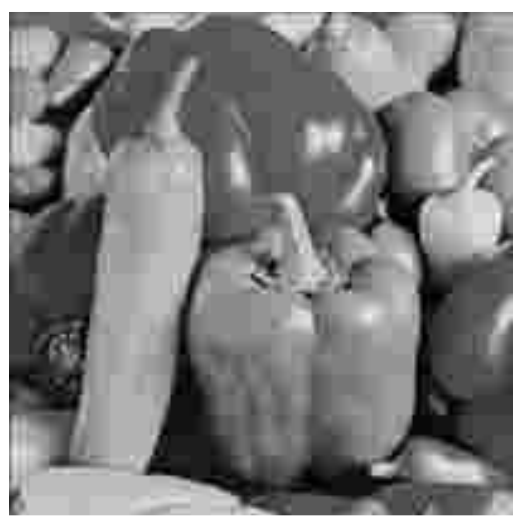

(a)
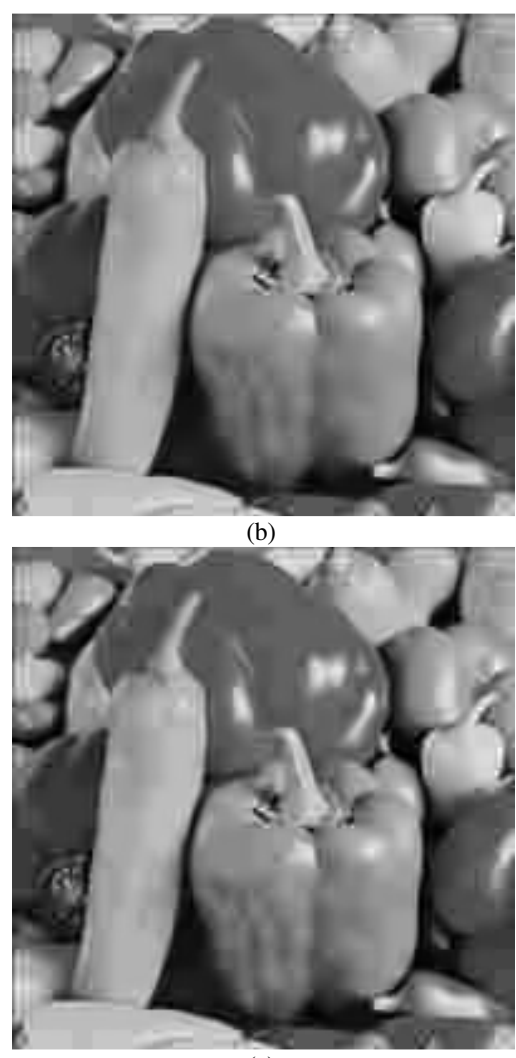

(c)

Figure 2. (a) Compressed "Peppers" image with $\mathrm{F}_{\mathrm{q}}=10$, Deblocked "Peppers" image using (b) Zhai method (c) Proposed method

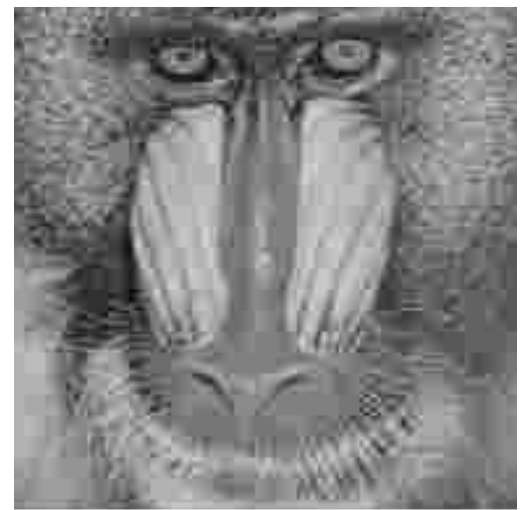

(a)

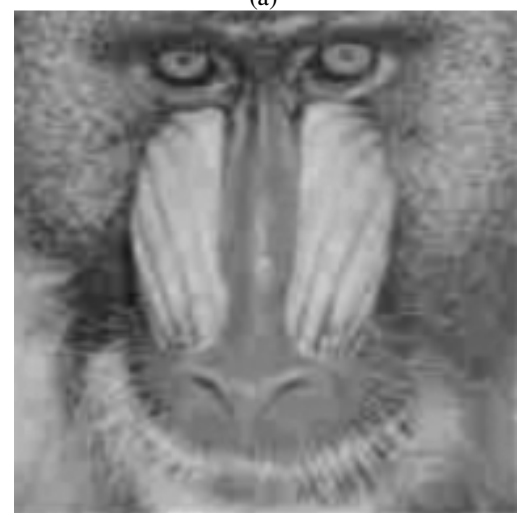

(b) 


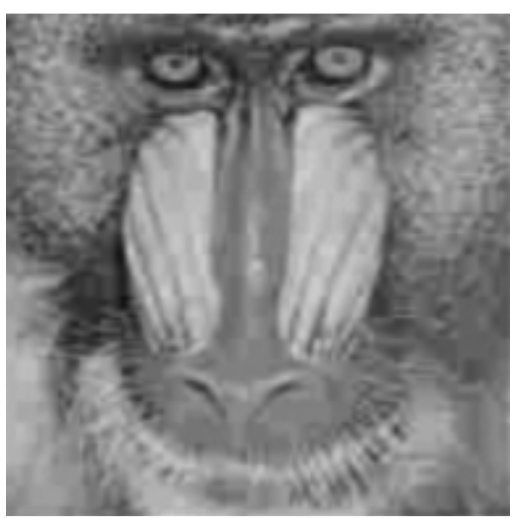

(c)

Figure 3. (a) Compressed "Baboon" image with $\mathrm{F}_{\mathrm{q}}=10$, Deblocked "Baboon" image using (b) Visibility map method (c) Proposed method
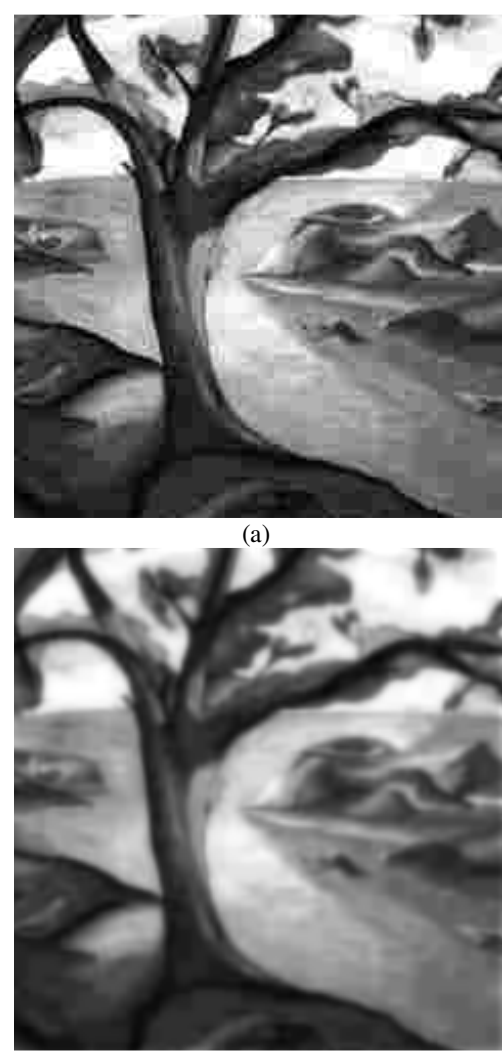

(b)

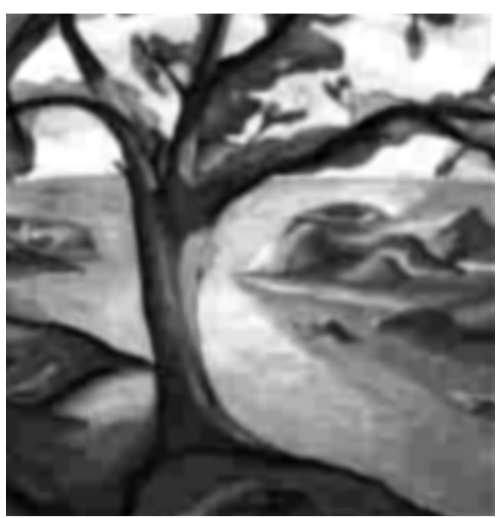

(c)

Figure 4. (a) Zoomed Compressed image with $\mathrm{F}_{\mathrm{q}}=10$ (b),(c) Zoomed zone corresponding to Fig 1(b) and (c), respectively. 